\title{
Acylated Cyanidin 3-sophoroside-5-glucosides from the Purple Roots of Red Radish (Raphanus sativus L.) 'Benikanmi'
}

\author{
Fumi Tatsuzawa ${ }^{1 *}$, Norio Saito ${ }^{2}$, Kenjiro Toki ${ }^{3}$, Koichi Shinoda $^{4}$, Atsushi Shigihara ${ }^{2}$ \\ and Toshio Honda ${ }^{2}$ \\ ${ }^{1}$ Laboratory of Olericultural and Floricultural Science, Faculty of Agriculture, Iwate University, Morioka, Iwate 020-8550, Japan \\ ${ }^{2}$ Faculty of Pharmaceutical Sciences, Hoshi University, Shinagawa, Tokyo 142-8501, Japan \\ ${ }^{3}$ Faculty of Horticulture, Minami-Kyushu University, Takanabe, Miyazaki 884-0003, Japan \\ ${ }^{4}$ National Agricultural Research Center for Hokkaido Region, Sapporo, Hokkaido 062-8555, Japan
}

Four new acylated cyanidin glycosides were isolated from the purple root peers of Raphanus sativus $\mathbf{L}$. 'Benikanmi', along with five known anthocyanins. These pigments were based on cyanidin 3-sophoroside-5glucoside, and acylated diversely with malonic, $p$-coumaric, caffeic, and ferulic acids. Two pigments of these four new anthocyanins were determined to be cyanidin 3- $O$-[2- $O$-( $\beta$-glucopyranosyl)-6- $O$-(trans-feruloyl)- $\beta$ glucopyranoside]-5- $O$-[6-O-(malonyl)- $\beta$-glucopyranoside] and cyanidin 3-[2-(glucosyl)-6-(cis- $p$-coumaroyl)glucoside]-5-[6-(malonyl)-glucoside] by chemical and spectroscopic methods. Since two other new pigments were obtained in small quantities, their structures were tentatively assigned to be malonyl cyanidin 3-sophoroside-5glucoside and malonyl cyanidin 3-[2-(glucosyl)-6-(trans-caffeoyl)-glucoside]-5-glucoside, mainly on the basis of their spectroscopic data. From the results, the potential of these purple root anthocyanins as natural food colorants is discussed.

Key Words: acylated cyanidin 3-sophoroside-5-glucoside, caffeic acid, ferulic acid, malonic acid, trans- and cis-pcoumaric acids.

\section{Introduction}

Recently, red radish (Raphanus sativus L.) root anthocyanins have been widely used as natural food colorant because they are potential alternatives to synthetic dyes (Giusti and Wrolstad, 2003). These anthocyanins have been identified by several research groups (Fuleki, 1969; Harborne, 1964; Ishikura and Hayashi, 1962, 1963, 1965; Ishikura et al., 1965), and the presence of pelargonidin and cyanidin derivatives was reported in red and purple roots of red radish cultivars, respectively. Moreover, several groups (Giusti et al., 1998; Mori et al., 2006; Otsuki et al., 2002; Tatsuzawa et al., 2008) characterized the structures of acylated pelargonidin glycosides from the red roots of red radish cultivars; however, a detailed structural study of the purple root anthocyanins of red radish cultivars has not been reported until now. In this study, we wish to report the structure elucidation of four new acylated

Received; July 10, 2009. Accepted; August 27, 2009.

* Corresponding author (E-mail: fumi@iwate-u.ac.jp). cyanidin 3-sophoroside-5-glucosides with five known anthocyanins isolated from purple root peels of Raphanus sativus 'Benikanmi'.

\section{Materials and Methods}

\section{General procedures}

TLC was carried out on plastic coated cellulose sheets (Merck) using nine mobile phases: $\mathrm{BAW}(n-\mathrm{BuOH} /$ $\left.\mathrm{HOAc} / \mathrm{H}_{2} \mathrm{O} .4: 1: 2, \mathrm{v} / \mathrm{v} / \mathrm{v}\right), \mathrm{BuHCl}(n-\mathrm{BuOH} / 2 \mathrm{~N} \mathrm{HCl}$, $1: 1$, v/v, upper layer), AHW $\left(\mathrm{HOAc} / \mathrm{HCl} / \mathrm{H}_{2} \mathrm{O}, 15: 3\right.$ : $82, \mathrm{v} / \mathrm{v} / \mathrm{v}), 1 \% \mathrm{HCl}$ and Forestal $\left(\mathrm{HOAc} / \mathrm{HCl} / \mathrm{H}_{2} \mathrm{O}, 30\right.$ : $3: 10, \mathrm{v} / \mathrm{v} / \mathrm{v})$ for anthocyanins, and BAW, APW (EtOAc/ pyridine $\left./ \mathrm{H}_{2} \mathrm{O}, 15: 7: 5, \mathrm{v} / \mathrm{v} / \mathrm{v}\right)$, EAA (EtOAc/HCOOH/ $\mathrm{H}_{2} \mathrm{O}, 5: 2: 1, \mathrm{v} / \mathrm{v} / \mathrm{v}$ ), APW (EtOAc/pyridine $/ \mathrm{H}_{2} \mathrm{O}, 15$ : $7: 5, \mathrm{v} / \mathrm{v} / \mathrm{v})$, and $15 \% \mathrm{HOAc}-\mathrm{H}_{2} \mathrm{O}$ for sugars and organic acids with UV light and aniline hydrogen phthalate spray regent (Tatsuzawa et al., 2006).

Analytical HPLC was performed using the LC 10A system (Shimadzu, Kyoto, Japan), using a Waters C18 $(4.6 \phi \times 250 \mathrm{~mm})$ column (Nihon Waters K.K., Tokyo, Japan) at $40^{\circ} \mathrm{C}$ with a flow rate of $1 \mathrm{~mL} \cdot \mathrm{min}^{-1}$ and monitoring at $530 \mathrm{~nm}$. The eluant was applied as a linear 
gradient elution for $40 \mathrm{~min}$ from 20 to $85 \%$ solvent $\mathrm{B}$ $\left(1.5 \% \mathrm{H}_{3} \mathrm{PO}_{4}, 20 \% \mathrm{HOAc}, 25 \% \mathrm{MeCN}\right.$ in $\left.\mathrm{H}_{2} \mathrm{O}\right)$ in solvent $\mathrm{A}\left(1.5 \% \mathrm{H}_{3} \mathrm{PO}_{4}\right.$ in $\left.\mathrm{H}_{2} \mathrm{O}\right)$. UV-Vis spectra were recorded on a MPS-2400 (Shimadzu) in $0.1 \% \mathrm{HCl}$ $\mathrm{MeOH}$ (from 200 to $700 \mathrm{~nm}$ ). Fast atom bombardment (FAB) mass spectra were obtained in the positive ion mode using the magic bullet (5:1 mixture of dithiothreitol and dithioerythritol) as a matrix. Nuclear magnetic resonance (NMR) spectra were recorded at $500 \mathrm{MHz}$ for ${ }^{1} \mathrm{H}$ spectra in DMSO- $d_{6}-\mathrm{CF}_{3} \mathrm{COOD}(9: 1)$. Chemical shifts are reported relative to a tetra methyl silane (TMS) internal standard $(\delta)$, and coupling constants $(J)$ are in Hz. Nuclear Overhauser enhancement spectroscopy (NOESY) spectra in DMSO- $d_{6}-\mathrm{CF}_{3} \mathrm{COOD}$ $(9: 1)$ at $21^{\circ} \mathrm{C}$ were recorded on a JMN LA-500 spectrometer (JEOL Ltd., Tokyo, Japan) using the pnoesy_fgzz method to obtain phase-sensitive data, with $2 \mathrm{k}$ complex data points in $\mathrm{f} 2$ and 512 increments in $\mathrm{f} 1$, $1100 \mathrm{~ms}$ mixing time (PI1). A $2.0 \mathrm{~s}$ delay before each scan was used and 80 scans were averaged.

\section{Plant materials}

Seeds of Raphanus sativus 'Benikanmi' (a cultivar of purple root) were purchased from Mikado Co., Ltd. (Chiba, Japan). These seeds were sown in September, 2006, and the plants were grown in a farm of Minamikyushu University. Their purple root peels [Purple 78A by Royal Horticultural Society (R.H.S.) Colour Chart and chromaticity value $\left.\left(\mathrm{b}^{*} / \mathrm{a}^{*}=-2.83 / 28.65=-0.10\right)\right]$ were collected in December, 2006, dried overnight at $40^{\circ} \mathrm{C}$, and kept in a refrigerator at about $4^{\circ} \mathrm{C}$.

\section{Extraction and purification of anthocyanins}

Dried root peels ( $c a .30 \mathrm{~g}$ ) of R. sativus 'Benikanmi' were immersed in $5 \% \mathrm{HOAc}-\mathrm{H}_{2} \mathrm{O}(5 \mathrm{~L})$ at room temperature for $5 \mathrm{~h}$ and extracted. The extract was passed through a Diaion HP-20 ion exchange resins (Mitsubishi Chemical, Tokyo, Japan) column $(90 \times 150 \mathrm{~mm})$, on which acylated anthocyanins were absorbed. The column was thoroughly washed with $\mathrm{H}_{2} \mathrm{O}(2 \mathrm{~L})$ and eluted $5 \%$ $\mathrm{HOAc}-\mathrm{MeOH}(500 \mathrm{~mL})$ to recover the anthocyanins. After concentration, the eluates were separated and purified with paper chromatography using BAW. The separated pigments were further purified with preparative HPLC, which was performed on a C18 (19 $\phi \times$ $150 \mathrm{~mm}$, Waters) column at $40^{\circ} \mathrm{C}$ with a flow rate of $1 \mathrm{~mL} \cdot \mathrm{min}^{-1}$ and monitoring at $530 \mathrm{~nm}$. The solvent used was as follows: a linear gradient elution for $55 \mathrm{~min}$ from 40 to $85 \%$ solvent B in solvent A. Finally, four new (14) and five known anthocyanins (A-E) (Fig. 1) were obtained as follows: pigments $\mathbf{1}$ (ca. $3 \mathrm{mg}), \mathbf{2}$ (ca. $3 \mathrm{mg}$ ), 3 (ca. $5 \mathrm{mg}), 4$ (ca. $10 \mathrm{mg}), \mathbf{A}(c a .1 \mathrm{mg}), \mathbf{B}(c a .1 \mathrm{mg})$, $\mathbf{C}(c a .1 \mathrm{mg}), \mathbf{D}(c a .1 \mathrm{mg})$, and $\mathbf{E}(c a .7 \mathrm{mg})$.

\section{Pigment 1}

Dark purple powder: malonyl cyanidin 3-sophoroside5 -glucoside; UV-VIS (in $0.1 \% \mathrm{HCl}-\mathrm{MeOH}$ ): $\lambda \max 524$, $279 \mathrm{~nm}, E_{440} / E_{\max }=15, \mathrm{AlCl}_{3}$ shift + ; TLC $R_{\mathrm{f}}$-values

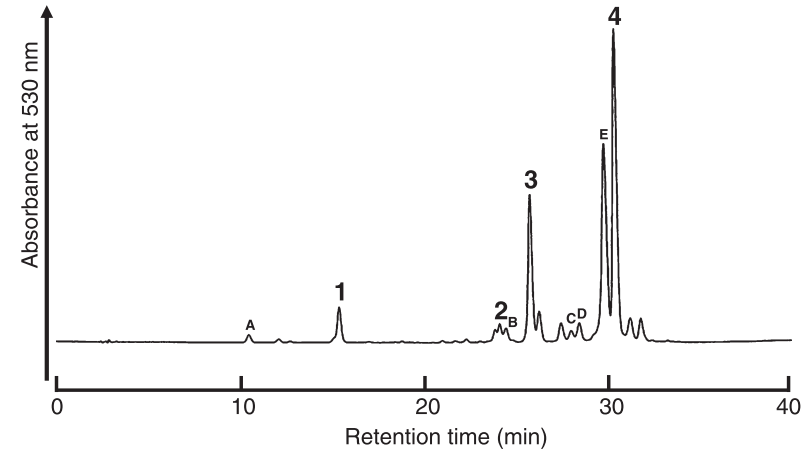

Fig. 1. HPLC analysis of pigments in root peels of $R$. sativus 'Benikanmi'. Peak No. 1-4 are new anthocyanins and A-E are known anthocyanins.

BAW 0.18, BuHCl 0.09, 1\% $\mathrm{HCl} 0.55$, AHW 0.78; HPLC: $R_{\mathrm{t}}(\min )$ 15.6.

\section{Pigment 2}

Dark purple powder: cyanidin 3-[2-(glucosyl)-6-(cis$p$-coumaroyl)-glucoside]-5-[6-(malonyl)-glucoside]; UV-VIS (in $0.1 \% \mathrm{HCl}-\mathrm{MeOH}$ ): $\lambda \max 529,318,296$, $282 \mathrm{~nm}, E_{\text {acyl }} / E_{\max }(\%)=106, E_{440} / E_{\max }=14, \mathrm{AlCl}_{3}$ shift + ; TLC $R_{\mathrm{f}}$-values BAW 0.39 , BuHCl $0.21,1 \% \mathrm{HCl} 0.43$, AHW 0.79; HPLC: $R_{\mathrm{t}}(\min ) 24.4$.

\section{Pigment 3}

Dark purple powder: malonyl cyanidin 3-[2(glucosyl)-6-(trans-caffeoyl)-glucoside]-5-glucoside; UV-VIS (in $0.1 \% \mathrm{HCl}-\mathrm{MeOH}$ ): $\lambda \max 527,328,295$, $280 \mathrm{~nm}, E_{\text {acyl }} / E_{\max }(\%)=74, E_{440} / E_{\max }=14, \mathrm{AlCl}_{3}$ shift +; TLC $R_{\mathrm{f}}$-values BAW $0.32, \mathrm{BuHCl} 0.16,1 \% \mathrm{HCl} 0.35$, AHW 0.60; HPLC: $R_{\mathrm{t}}(\mathrm{min}) 26.0$.

\section{Pigment 4}

Dark purple powder: cyanidin 3-O-[2-O-( $\beta$-glucopyranosyl)-6- $O$-(trans-feruloyl)- $\beta$-glucopyranoside]-5- $O$ [6-O-(malonyl)- $\beta$-glucopyranoside]; UV-VIS (in $0.1 \%$ $\mathrm{HCl}-\mathrm{MeOH}): \lambda \max 530,323,295,281 \mathrm{~nm}, E_{\text {acyl }} / E_{\max }$ $(\%)=88, E_{440} / E_{\max }=14, \mathrm{AlCl}_{3}$ shift + ; TLC $R_{\mathrm{f}}$-values BAW 0.36, BuHCl 0.16, $1 \% \mathrm{HCl} 0.43$, AHW 0.67; HPLC: $R_{\mathrm{t}}(\min ) 30.6$.

\section{Pigment $A$}

Dark purple powder: cyanidin 3-sophoroside-5glucoside; UV-VIS (in $0.1 \% \mathrm{HCl}-\mathrm{MeOH}$ ): $\lambda \max 525$, $278 \mathrm{~nm}, E_{440} / E_{\max }=13, \mathrm{AlCl}_{3}$ shift + ; TLC $R_{\mathrm{f}}$-values BAW 0.20, BuHCl 0.06, $1 \% \mathrm{HCl} 0.46$, AHW 0.72; HPLC: $R_{\mathrm{t}}(\min ) 10.5$.

\section{Pigment $B$}

Dark purple powder: cyanidin 3-[2-(glucosyl)-6-(transcaffeoyl)-glucoside]-5-glucoside; UV-VIS (in $0.1 \%$ $\mathrm{HCl}-\mathrm{MeOH}): \lambda \max 528,327,294,281 \mathrm{~nm}, E_{\text {acyl }} / E_{\max }$ $(\%)=83, E_{440} / E_{\max }=15, \mathrm{AlCl}_{3}$ shift + ; TLC $R_{\mathrm{f}}$-values BAW 0.40, BuHCl 0.17, $1 \% \mathrm{HCl} 0.28$, AHW 0.57; HPLC: $R_{\mathrm{t}}(\min ) 24.6$.

\section{Pigment $C$}

Dark purple powder: cyanidin 3-[2-(glucosyl)-6(trans-p-coumaroyl)-glucoside]-5-glucoside; UV-VIS (in $0.1 \% \mathrm{HCl}-\mathrm{MeOH}$ ): $\lambda \max 528,(314), 297,282 \mathrm{~nm}$, 
$E_{\text {acyl }} / E_{\max }(\%)=160, E_{440} / E_{\max }=15, \mathrm{AlCl}_{3}$ shift +; TLC $R_{\mathrm{f}}$-values BAW 0.44, BuHCl 0.20, 1\% HCl 0.31, AHW 0.62; HPLC: $R_{\mathrm{t}}(\min ) 28.2$.

\section{Pigment $D$}

Dark purple powder: cyanidin 3-[2-(glucosyl)-6(trans-feruloyl)-glucoside]-5-glucoside; UV-VIS (in $0.1 \% \mathrm{HCl}-\mathrm{MeOH}): \lambda \max 529,323,295,281 \mathrm{~nm}, E_{\text {acyl }} /$ $E_{\max }(\%)=86, E_{440} / E_{\max }=14, \mathrm{AlCl}_{3}$ shift +; TLC $R_{\mathrm{f}^{-}}$ values BAW $0.40, \mathrm{BuHCl} 0.15,1 \% \mathrm{HCl} 0.30$, AHW 0.60; HPLC: $R_{\mathrm{t}}(\min ) 28.6$.

\section{Pigment $E$}

Dark purple powder: cyanidin 3-[2-(glucosyl)-6(trans- $p$-coumaroyl)-glucoside]-5-[6-(malonyl)-glucoside]; UV-VIS (in $0.1 \% \mathrm{HCl}-\mathrm{MeOH}$ ): $\lambda \max 529,315$, 296, $281 \mathrm{~nm}, E_{\text {acyl }} / E_{\max }(\%)=76, E_{440} / E_{\max }=13, \mathrm{AlCl}_{3}$ shift +; TLC $R_{\mathrm{f}}$-values BAW $0.40, \mathrm{BuHCl} 0.27,1 \% \mathrm{HCl}$ 0.45, AHW 0.72; HPLC: $R_{\mathrm{t}}(\min ) 30.0$.

\section{Results and Discussion}

Five known anthocyanins (A-E) from these anthocyanin peaks (Fig. 1) were isolated, and their structures were determined to be cyanidin 3-sophoroside-5glucoside (ranging from $0.8 \%$ of the total anthocyanin contents calculated from the HPLC peak area at $530 \mathrm{~nm}, \mathbf{A})$, cyanidin 3-[2-(glucosyl)-6-(trans-caffeoyl)-glucoside]-5glucoside $(1.7 \%, \mathbf{B})$, cyanidin 3-[2-(glucosyl)-6-(trans-pcoumaroyl)-glucoside]-5-glucoside $(1.1 \%, \mathbf{C})$, cyanidin 3-[2(glucosyl)-6-(trans-feruloyl)-glucoside]-5-glucoside (1.8\%, D), and cyanidin 3-[2-(glucosyl)-6-(trans-p-coumaroyl)glucoside]-5-[6-(malonyl)-glucoside] $(26.8 \%, \mathbf{E})$ by direct comparison with authentic specimens (Idaka et al., 1987; Saito et al., 1995, 2008). In addition to the above five anthocyanins, four new anthocyanins (1: $3.8 \%, 2: 1.1 \%$, 3: $15.4 \%, 4: 39.9 \%$ ) were detected in the extract as shown in Figure 1. Acid hydrolysis of each pigment 14 (ca. $0.3 \mathrm{mg}$ each) was carried out with $2 \mathrm{~N} \mathrm{HCl}(1 \mathrm{~mL})$ at $90^{\circ} \mathrm{C}$ for $2 \mathrm{~h}$ to give cyanidin, glucose, and malonic acid. $c i s-p$-Coumaric acid, caffeic acid, and ferulic acid were detected in the hydrolysis products of pigments 2 , $\mathbf{3}$, and 4, respectively. These products were confirmed by direct comparison of their TLC and HPLC behavior with authentic samples. Alkaline hydrolysis of pigments 1-4 (ca. $0.3 \mathrm{mg}$ ) was carried out with $2 \mathrm{~N} \mathrm{NaOH}$ solution $(0.5 \mathrm{~mL})$ at ambient temperature for $15 \mathrm{~min}$, and the mixture was then acidified with $2 \mathrm{~N} \mathrm{HCl}(0.7 \mathrm{~mL})$ to provide only one deacylated anthocyanin. Its structure was identified to be cyanidin 3-sophoroside-5-glucoside by direct comparison of its TLC and HPLC behavior with those of the authentic sample obtained from Iberis umbellata (Saito et al., 2008). Also on acyl residues, malonic acid was detected in alkaline hydrolysis products of 1-4, cis- $p$-coumaric acid in $\mathbf{2}$, caffeic acid in $\mathbf{3}$, and ferulic acid in $\mathbf{4}$, respectively.

The FAB mass spectra of pigments $\mathbf{1 - 4}$ gave molecular ions at 859, 1005, 1021 and $1035 \mathrm{~m} / \mathrm{z}$, respectively, in agreement with the masses calculated for $\mathrm{C}_{36} \mathrm{H}_{43} \mathrm{O}_{24}, \mathrm{C}_{45} \mathrm{H}_{49} \mathrm{O}_{26}, \mathrm{C}_{45} \mathrm{H}_{49} \mathrm{O}_{27}$ and $\mathrm{C}_{46} \mathrm{H}_{51} \mathrm{O}_{27}$, and their elemental components were confirmed by measuring their high-resolution FAB MS; Pigment 1: calc. for $\mathrm{C}_{36} \mathrm{H}_{43} \mathrm{O}_{24}$ requires: 859.2144. Found: 859.2147; Pigment 2: calc. for $\mathrm{C}_{45} \mathrm{H}_{49} \mathrm{O}_{26}$ requires: 1005.2512. Found: 1005.2542; Pigment 3: calc. for $\mathrm{C}_{45} \mathrm{H}_{49} \mathrm{O}_{27}$ requires: 1021.2461. Found: 1021.2467; Pigment 4: calc. for $\mathrm{C}_{46} \mathrm{H}_{51} \mathrm{O}_{27}$ requires: 1035.2616. Found: 1035.2603. Structure elucidation of pigment 4 was further carried out by measuring its ${ }^{1} \mathrm{H}-{ }^{1} \mathrm{H}$ correlation spectroscopy (COSY), nuclear Overhauser enhancement and exchange spectroscopy (NOESY), heteronuclear multiple quantum coherence spectroscopy (HMQC) and heteronuclear multiple bond correlation spectroscopy (HMBC) spectra, and its chemical shifts are shown in Table 1

\section{Pigment 4}

The ${ }^{1} \mathrm{H}$ NMR spectrum of pigment 4 demonstrated the presence of three molecules of glucose and one molecule each of cyanidin, ferulic acid, and malonic acid. The aromatic protons of cyanidin and ferulic acid in this pigment were assigned by analysis of the COSY spectrum (Table 1). Proton signals of the sugar moieties were observed in the region of $\delta 2.74-5.68$ (Table 1 ). The signals of three anomeric protons appeared at $\delta 5.68$ $(1 \mathrm{H}, d, J=7.3 \mathrm{~Hz}$, glucose (Glc) A), $\delta 4.70(1 \mathrm{H}, d, J=$ $7.6 \mathrm{~Hz}, \mathrm{Glc} \mathrm{B})$ and $\delta 5.18(1 \mathrm{H}, d, J=7.9 \mathrm{~Hz}$, Glc C), and the assigned neighboring diaxial hydrogens had large coupling constants $(7.3-9.2 \mathrm{~Hz})$; therefore, these three glucose units must be $\beta$-glucopyranose. The characteristic four protons shifted to lower magnetic fields were assigned to two methylenes $\left(-\mathrm{CH}_{2}-\right)$ of Glc $\mathrm{A}(\delta 4.28$ and 4.47) and Glc $\mathrm{C}(\delta 3.94$ and 4.41). These results revealed that the OH- 6 of Glc $\mathrm{A}$ and $\mathrm{C}$ were acylated by two acid residues (ferulic and malonic acid). Moreover, a signal appeared at $\delta 4.12(t, \mathrm{~J}=7.8 \mathrm{~Hz}, \mathrm{H}$ 2 of Glc A) was easily correlated to the proton $\mathrm{H}-1$ of Glc A. Thus, this proton was assigned to the $\mathrm{H}-2$ of Glc A. The signal of the anomeric proton of Glc B was correlated to the signal of the $\mathrm{H}-2$ proton of Glc A in the NOESY spectrum. These results suggested that Glc $\mathrm{B}$ was attached to $\mathrm{OH}-2$ of Glc A through a glucosidic bond, and formed a sophorose unit. The signals of anomeric protons of Glc A and Glc $\mathrm{C}$ were correlated to the signals of the H-4 and H-6 of cyanidin by analysis of NOESY spectra (Fig. 2). Moreover, the observed correlation between the methylene protons of Glc $\mathrm{C}$ and malonic acid in its NOESY spectrum indicated that malonic acid was bonded to the OH-6 of Glc C. On the hydrolysis of pigment $4(1 \mathrm{mg})$ with $1 \mathrm{~N} \mathrm{HCl}(1 \mathrm{~mL})$ at room temperature for 1 week, pigment $\mathrm{D}$ was yielded; therefore, pigment $\mathbf{4}$ was determined to be cyanidin 3-O-[2-O-( $\beta$-glucopyranosyl)-6-O-(trans-feruloyl)- $\beta$ glucopyranoside]-5- $O$-[6- $O$-(malonyl)- $\beta$-glucopyranoside $]$ (Fig. 2), a novel anthocyanin in plants (Andersen and Jordheim, 2006; Harborne and Baxter, 1999; Honda and Saito, 2002). 
Table 1. ${ }^{1} \mathrm{H}$ and ${ }^{13} \mathrm{C} \mathrm{NMR}^{2}$ spectroscopic data of pigment 4 of $R$. sativus 'Benikanmi'.

\begin{tabular}{|c|c|c|c|c|c|}
\hline & ${ }^{1} \mathrm{H}$ & ${ }^{13} \mathrm{C}$ & & ${ }^{1} \mathrm{H}$ & ${ }^{13} \mathrm{C}$ \\
\hline Cyanidin & & & Glucose C & & \\
\hline 2 & & 162.6 & 1 & $5.18 \mathrm{~d}(7.9)$ & 101.7 \\
\hline 3 & & 144.7 & 2 & $3.55 \mathrm{t} *(8.5)$ & 73.2 \\
\hline 4 & $8.77 \mathrm{~s}$ & 131.3 & 3 & $3.41 \mathrm{t} *(8.9)$ & 76.1 \\
\hline 5 & & 154.9 & 4 & $3.19 \mathrm{~m}$ & 69.7 \\
\hline 6 & $6.97 \mathrm{~d}(1.6)$ & 104.7 & 5 & $3.76 \mathrm{~m}$ & 74.2 \\
\hline 7 & & 167.3 & $6 a$ & $3.94 \mathrm{dd}(7.0,12.0)$ & 64.2 \\
\hline 8 & $7.05 \mathrm{~d}(1.6)$ & 96.3 & $6 b$ & $4.41 \mathrm{~d}(12.0)$ & \\
\hline 9 & & 155.2 & & & \\
\hline 10 & & 111.5 & Ferulic acid & & \\
\hline $1^{\prime}$ & & 119.7 & 1 & & 125.5 \\
\hline $2^{\prime}$ & $8.05 \mathrm{~d}(2.2)$ & 117.7 & 2 & $7.07 \mathrm{~d}(1.6)$ & 111.3 \\
\hline $3^{\prime}$ & & 146.4 & 3 & & 147.9 \\
\hline $4^{\prime}$ & & 155.1 & 4 & & 149.5 \\
\hline $5^{\prime}$ & $7.10 \mathrm{~d}(8.6)$ & 117.0 & 5 & $6.75 \mathrm{~d}(8.3)$ & 115.5 \\
\hline \multirow[t]{2}{*}{$6^{\prime}$} & $8.27 \mathrm{dd}(2.2,8.6)$ & 127.8 & 6 & $6.96 \mathrm{dd}(1.6,8.3)$ & 123.1 \\
\hline & & & $7 \alpha$ & $6.33 \mathrm{~d}(15.9)$ & 114.5 \\
\hline Glucose A & & & $8 \beta$ & $7.42 \mathrm{~d}(15.9)$ & 145.6 \\
\hline 1 & $5.68 \mathrm{~d}(7.3)$ & 99.2 & 9 & & 166.8 \\
\hline 2 & $4.12 \mathrm{t} *(7.8)$ & 80.8 & $\mathrm{OCH}_{3}$ & $3.74 \mathrm{~s}$ & 55.7 \\
\hline 3 & $3.74 \mathrm{t} *(8.2)$ & 76.2 & & & \\
\hline 4 & $3.52 \mathrm{t} *(9.2)$ & 69.7 & Malonic acid & & \\
\hline 5 & $4.03 \mathrm{~m}$ & 73.7 & 1 & & 166.9 \\
\hline $6 a$ & $4.28 \mathrm{dd}(6.6,12.0)$ & 63.2 & 2 & $3.41 \mathrm{~s}$ & 41.2 \\
\hline $6 b$ & $4.47 \mathrm{~d}(12.0)$ & & 3 & & 168.1 \\
\hline \multicolumn{6}{|l|}{ Glucose B } \\
\hline 1 & $4.70 \mathrm{~d}(7.6)$ & 103.8 & & & \\
\hline 2 & $3.00 \mathrm{t} *(8.9)$ & 74.6 & & & \\
\hline 3 & $3.12 \mathrm{t} *(8.9)$ & 75.8 & & & \\
\hline 4 & $3.04 \mathrm{t} *(9.2)$ & 69.7 & & & \\
\hline 5 & $2.74 \mathrm{~m}$ & 76.9 & & & \\
\hline $6 a$ & $3.19 \mathrm{~m}$ & 60.6 & & & \\
\hline $6 \mathrm{~b}$ & $3.23 \mathrm{~m}$ & & & & \\
\hline
\end{tabular}

${ }^{z} 125.78 \mathrm{MHz}$ for ${ }^{13} \mathrm{C}$ and $500 \mathrm{MHz}$ for ${ }^{1} \mathrm{H}\left(\mathrm{DMSO}-d_{6}-\mathrm{CF}_{3} \mathrm{CO}_{2} \mathrm{D}, 9: 1\right)$, at $25^{\circ} \mathrm{C}$, TMS as an internal standard. Coupling constants $(J$ in $\mathrm{Hz})$ in parentheses.

$\mathrm{s}=$ singlet, $\mathrm{d}=$ doublet, brd $=$ broad doublet, $\mathrm{t}^{*}=$ distorted triplet, $\mathrm{m}=$ multiplet, $\mathrm{dd}=$ double doublet.

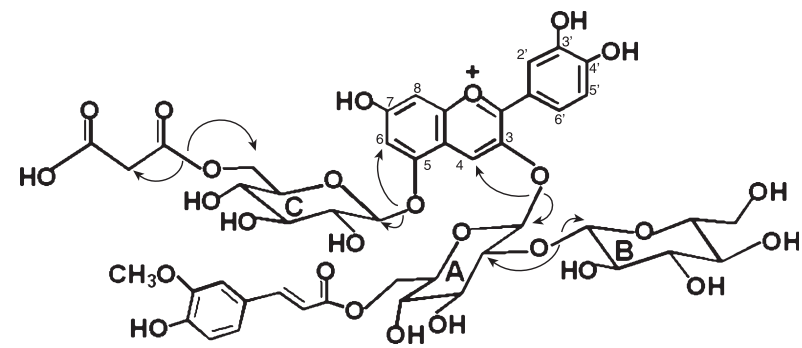

Fig. 2. Novel acylated anthocyanin (pigment 4) from root peels of Raphanus sativus 'Benikanmi'. Observed NOEs are indicated by arrows.

\section{Pigments 1-3}

Pigments 1-3 were hydrolyzed with $1 \mathrm{~N} \mathrm{HCl}$ by several methods, or isomerized by sunlight (Tatsuzawa et al., 2008). Pigments 1 (1 mg) and $\mathbf{3}(1 \mathrm{mg})$ were hydrolyzed with $1 \mathrm{~N} \mathrm{HCl}(1 \mathrm{ml})$ at room temperature for 1 week. Then, pigment $\mathbf{A}$ from pigment $\mathbf{1}$ and pigment $\mathbf{B}$ from pigment 3 (Fig. 1) were obtained in addition to malonic acid as their hydrolysates, respectively; therefore, pigments $\mathbf{1}$ and $\mathbf{3}$ were tentatively identified to be malonyl cyanidin 3-sophorosie-5-glucoside (pigment 1) and malonyl cyanidin 3-[2-(glucosyl)-6-(transcaffeoyl)-glucoside]-5-glucoside (pigment 3), respectively. Pigment $\mathbf{2}$ was transformed into pigment $\mathbf{C}$ by isomerization with sunlight (Tatsuzawa et al., 2008), indicating that the $p$-coumaroyl moiety of pigment 2 was isomerized from cis to trans form, and its structure was confirmed by HPLC analysis by comparison with pigment $\mathbf{C}$. Therefore, pigment $\mathbf{2}$ was identified to be cyanidin 3-[2-(glucosyl)-6-(cis-p-coumaroyl)glucoside]-5-malonylglucoside.

To the best of our knowledge, acylated anthocyanins 
have been characterized by several research groups for red-color root cultivars whose anthocyanins were based on pelargonidin 3-sophoroside-5-glucoside (Giusti et al., 1998; Mori et al., 2006; Otsuki et al., 2002; Tatsuzawa et al., 2008); however, there are no reports on the anthocyanin components of purple-color root cultivars until now except the classical reports of Ishikura and Hayashi (1965) and Ishikura et al., (1965). In this study, we made a planed to investigate the anthocyanin components of 'Benikanmi', a typical purple-color root cultivar of red radish. The results revealed that the anthocyanins of 'Benikanmi' were analogous to those of red-color root cultivars, except for their aglycones. Namely, the aglycone of 'Benikanmi' is cyanidin instead of pelargonidin for red-color root cultivars. Therefore, it was considered that anthocyanin pigments from purplecolor root cultivars of red radish also have potential as natural food colorants purple or red-purple for drinks, candy, jelly and so on. We hope that this anthocyanin research will contribute to the development of natural food colorants in the future.

\section{Acknowledgement}

We thank Mr. James Hall (Iwate University) for careful revision of the manuscript.

\section{Literature Cited}

Andersen, Ø. M. and M. Jordheim. 2006. The anthocyanins. p. 471-551. In: Ø. M. Andersen, and K. R. Markham, (eds.). Flavonoids: Chemistry, Biochemistry and Applications. CRC Press, Boca Raton.

Fuleki, T. 1969. The anthocyanins of strawberry, rhubarb, radish and onion. J. Food Sci. 34: 365-269.

Giusti, M. M. and R. E. Wrolstad. 2003. Acylated anthocyanins from edible source and their applications in food systems. Biochem. Eng. J. 14: 217-225.

Giusti, M. M., H. Ghanadan and R. E. Wrolstad. 1998. Elucidation of the structure and conformation of red radish (Raphanus sativus) anthocyanins using one- and two-dimentional nuclear magnetic resonance techniques. J. Agric. Food Chem. 46: $4858-4863$.

Harborne, J. B. 1964. Plant polyphenols-XI. The structure of acylated anthocyanins. Phytochemistry 3: 151-160.
Harborne, J. B. and H. Baxter. 1999. The Handbook of Natural Flavonoids, vol. 2. p. 1-114. John Wiley \& Sons, Chichester.

Honda, T. and N. Saito. 2002. Recent progress in the chemistry of polyacylated anthocyanins as flower color pigments. Heterocycles 56: 633-692.

Idaka, E., K. Suzuki, H. Yamakita, T. Ogawa, T. Kondo and T. Goto. 1987. Structure of monoacylated anthocyanins isolated from red cabbage, Brassica oleracea. Chemistry Letters: 145 148.

Ishikura, N. and K. Hayashi. 1962. Anthocyanins in red roots of a radish. Studies on anthocyanins, XXXVI. Bot. Mag. Tokyo 75: $28-36$.

Ishikura, N. and K. Hayashi. 1963. Chromatographic separation and characterization of the component anthocyanins in radish root. Study on anthocyanins, XXXVIII. Bot. Mag. Tokyo 76: $6-13$.

Ishikura, N. and K. Hayashi. 1965. Separation and identification of the complex anthocyanins in purple radish studies on anthocyanins, XLVI. Bot. Mag. Tokyo 78: 91-96.

Ishikura, N., T. Hoshi and K. Hayashi. 1965. Crystallization and characterization of the basic triglucosides common to all components in purple pigment of hybrid radish studies on anthocyanins, XLV. Bot. Mag. Tokyo 78: 8-13.

Mori, M., S. Nakagawa, M. Maeshima, S. Niikura and K. Yoshida. 2006. Anthocyanins from the rhizome of Raphanus sativus, and change in the composition during maturation. Heterocycles 69: 239-251.

Otsuki, T., H. Matsufuji, M. Takeda, M. Toyoda and Y. Goda. 2002. Acylated anthocyanins from red radish (Raphanus sativus L.). Phytochemistry 60: 79-87.

Saito, N., F. Tatsuzawa, E. Suenaga, K. Toki, K. Shinoda, A. Shigihara and T. Honda. 2008. Tetra-acylated cyanidin 3sophoroside-5-glucosides from the flowers of Iberis umbellata L. (Cruciferae). Phytochemistry 69: 3139-3150.

Saito, N., F. Tatsuzawa, K. Yoda, M. Yokoi, K. Kasahara, S. Iida, A. Shigihara and T. Honda. 1995. Acylated cyanidin glycosides in the violet-blue flowers of Ipomoea purpurea. Phytochemistry 40: 1283-1289.

Tatsuzawa, F., N. Saito, K. Shinoda, A. Shigihara and T. Honda. 2006. Acylated cyanidin 3-sambubioside-5-glucosides in three garden plants of the Cruciferae. Phytochemistry 67: $1287-1295$.

Tatsuzawa, F., K. Toki, N. Saito, K. Shinoda, A. Shigihara and T. Honda. 2008. Anthocyanin occurrence in the root peels, petioles and flowers of red radish (Raphanus sativus L.). Dyes and pigments 79: 83-88. 